\title{
Co-learning of Functions by Probabilistic Algorithms
}

\author{
Ilja Kucevalovs, Kaspars Balodis and Rūsiņš Freivalds \\ Institute of Mathematics and Computer Science \\ University of Latvia \\ Riga, Latvia \\ Rusins.Freivalds@mii.lu.lv
}

\begin{abstract}
We investigate properties of an identification type of recursive functions, called co-learning. The inductive process refutes all possible programs but one, and, by definition, this program is demanded to be correct. This type of identification was introduced in [6]. M. Kummer in the paper [9] showed that this type characterizes computable numberings possessing a certain property thus answering a long standing open problem by Yu. L. Ershov [2]. We consider probabilistic algorithms of co-learning and establish an infinite discrete hierarchy of classes of recursive functions. The parameters of this new hierarchy coincide with the hierarchy by R. Freivalds [4] for probabilistic algorithms of finite identification.
\end{abstract}

Keywords-inductive inference; co-learning; probabilistic algorithms

\section{INTRODUCTION}

Graphs of total recursive functions, i. e. the sets

$$
G_{f}=\{(x, f(x)) \mid x \in \mathrm{N}\}
$$

are recursive sets. Hence they are recursively enumerable and co-recursively enumerable (complements of recursively enumerable sets).

Theorem 3 in [10] section 4.4 shows that, if the graph of a total function $f$ is recursively enumerable, then $f$ is recursive.

The inverse theorem does not hold.

The paper [3] contains the following generalization of this assertion.

Theorem 1. There is a total function $f$ such that:

1. the graph off is co-recursively enumerable,

2. for arbitrary partial recursive function $g$, the intersection of the graphs of $f$ and $g$ is no more than finite.

The idea used in this proof has led to introduction of the following definition in the paper [6]:

Definition 1. Let $\psi$ be a numbering containing at least one index for every function $f$ in the class $U$. We say that the class $U$ is co-learnable $(U \in \operatorname{co}-F I N(\psi))$ if, for arbitrary $f \in U$, the inductive inference machine outputs an infinite sequence of integers, and the lacking one is a correct $\psi$ index for the function $f$ to be learned.

This definition was used in [6] for various different numberings. Recursively enumerable classes of total recursive functions and recursively enumerable numberings of total recursive functions were considered (in order to escape from using many-words terms, we will refer to them as $\tau$-numberings). Gödel numberings are the most practical numberings of the class of all the partial recursive functions because they correspond to universal programming languages. However other computable numberings of the class of all the partial recursive functions were considered as well.

This definition of co-learnability is not so unexpected. There exist many practical learning algorithms that attempt to find an object with specific properties from a finite set. This set is defined by practical needs. Always it is large and exhaustive search is impossible because of time restrictions. These algorithms used gradually eliminate large families of these formulae up to a moment when only one formula is left. In our terms, this kind of algorithms is a co-learning in a finite space.

We have used the notions and the standard notation in inductive inference without detailed explanations. See [1] and [2] for references. The notions recursion theory are described in much detail in the monographs $[11,12]$.

We use the following shortenings. By $E X$ we denote the family of classes of total recursive functions identifiable in the limit. FIN denotes the family of classes of total recursive functions finitely identifiable. $f^{[y]}$ is the initial fragment $f(0), f(1), \ldots, f(y)$ of the function $f$.

\section{CO-LEARNING IN RECURSIVELY ENUMERABLE NUMBERINGS}

We consider the most natural classes of total recursive functions, called recursively enumerable classes. These classes are characterized by existence of a governing mechanism for this class, called universal function. This mechanism plays a role of a programming language.

Formally, a class $U$ of total recursive functions is called recursively enumerable if there is a total recursive 2argument function $\tau(n, x)$ such that $U=\left\{\tau_{0}, \tau_{1}, \tau_{2}, \ldots\right\}$ where $\tau_{i}(x)=\lambda x . \tau(i, \mathrm{x})$.

In effect, such a function $\tau$ presents a numbering of the functions in $U$. The first index $i$ may be considered as a program and the second index $x$ may be considered as the input data.

In the existing literature these numberings are usually called "recursively enumerable numberings". To make our text shorter, we will call them $\tau$-numberings. 
The same class $U$ can have many different $\tau$-numberings. Usually, it is believed that not much depends on the programming language. We will show below that in many cases co-learnability of a class $U$ depends of the specific $\tau$ numbering crucially. below.

We list some most important results from [6] and [8]

Theorem 2. Let an arbitrary recursively enumerable class $U$ of total recursive functions be given Then there exists an recursively enumerable numbering $\tau$ of $U$ such that $U \in \operatorname{co}-\operatorname{FIN}(\tau)$

Theorem 3. There are a recursively enumerable class $U$ of total recursive functions and a recursively enumerable numbering $\tau$ of this class $U$ such that $U \notin c o-F I N(\tau)$.

Definition 2. Let $U$ be a class $U$ of total recursive functions. A function $f$ such that, for arbitrary $x$, there is a function $g \neq f, g^{[x]}=f^{[x]}$ and $g \in U$, is called an accumulation point of the class $U$.

Theorem 4. Let $U$ be a class $U$ of totally definedl recursive functions. If $U$ contains at least one accumulation point, then there exists a recursively enumerable numbering $\tau$ of the class $U$ such that $U \notin c o-F I N(\tau)$.

We end this listing of preceding results by citing the paper by R. Freivalds, D. Gobleja, M. Karpinski and C.H. Smith [5] where a surprising result was shown

Theorem 5. There is a recursively enumerable class $A$ of total recursive functions such that:

1. A is co-learnable in all recursively enumerable numberings $\tau$ of $A$,

2. A is not FIN-identifiable.

Later M. Kummer [9] proved important theorem with far-reaching consequences.

Theorem 6. The following properties of recursively enumerable classes of total recursive functions are equivalent:

1. A is co-learnable in all recursively enumerable numberings $\tau$ of $A$,

2. all recursively enumerable numberings $\tau$ of $A$ are reducible one to another.

Based on this Theorem, he solved a long-standing problem by Yu.L.Ershov. We consider this fact as one more proof of the naturality of the notion of co-learnability.

\section{PROBABILISTIC CO-LEARNING IN RECURSIVELY ENUMERABLE NUMBERINGS}

Definition 3. Let $T$ be a (finite or infinite) team of inductive inference machines. We say that a class $U$ of total recursive functions is co-learned by $T$ of for arbitrary function $f \in U$ there is at least one inductive inference machine in the team $T$ which co-learns $f$ correctly.

Theorem 7. There exists a class $U$ of total recursive functions such that:

1. for arbitrary recursively enumerable numbering $\tau$ of the class $U$, there is a team of two inductive inference machines co-learning $U$ in the numbering $\tau$.

2. there is a recursively enumerable numbering $\tau$ such that no single inductive inference machine can colearn $U$ in the numbering $\tau$.
Probabilistic learning and probabilistic identification are similar to but different from team learning and team identification.

Definition 4. We say that a class $U$ of total recursive functions is co-learned by a probabilistic inductive inference machine with a probability $p$ if for arbitrary function $f \in U$ the probability of outputting a correct result is at least $p$.

Notice that in this definition no randomization of target functions is allowed. Randomization refers only to the process of computation.

For rather many models of inductive inference team identifiability (learnability) is equivalent to probabilistic identifiability (learnability). Fortunately or not, but colearning is different. The class $U$ in the proof of Theorem 7 cannot be probabilistically co-learned. However a counterpart of Theorem 7 can be proved.

Theorem 8. There exists a class $V$ of total recursive functions such that:

1. for arbitrary recursively enumerable numbering $\tau$ of the class $V$, there exists a probabilistic inductive inference machine co-learning $V$ in the numbering $\tau$ with the probability $\frac{2}{3}$

2. there is a recursively enumerable numbering $\tau$ such that no single deterministic inductive inference machine can co-learn $V$ in the numbering $\tau$.

The considered proof can be extended to prove the following theorem.

Theorem 9. There exists a class $V$ consisting of total recursive functions such that $V$ is characterized by two properties:

1. if $\tau$ is an arbitrary recursively enumerable numbering of the class $V$, then there is a probabilistic inductive inference machine co-learning $V$ in the numbering $\tau$ with the probability $\frac{m}{n}$.

2. there exists a recursively enumerable numbering $\tau$ such that no single deterministic inductive inference machine can co-learn $V$ in the numbering $\tau$.

Co-learnability of recursively enumerable classes of total recursive functions is very similar to the FIN-identifiability. However our

Theorem 9 shows a sharp distinction with the step-wise hierarchy of probabilistic finite identifiability proved by $\mathrm{R}$. Freivalds [4].

\section{ACKNOWLEDGMENT}

Our research was financed by Project 271/2012 from the Latvian Council of Science and supported by the European Social Fund within the project «Support for Doctoral Studies at University of Latvia».

\section{REFERENCES}

[1] D. Angluin and C. Smith. Inductive inference: Theory and methods. Computing Surveys, 15:237-269, 1983.

[2] Yu. L. Ershov. Theory of numberings. Nauka, Moscow, 1977 (Russian).

[3] R. Freivalds. Minimal Gödel numbers and their identification in the limit. Lecture Notes in Computer Science, Springer, 32: 219-225, 1975. 
[4] R. Freivalds. Finite identification of general recursive functions by probabilistic strategies. Proc. 2nd International Conference " Fundamentals of Computation Theory ", Berlin, Akademie, 138-145, 1979.

[5] R. Freivalds, D. Gobleja, M. Karpinski and C.H. Smith. Colearnability and FIN-identifiability of enumerable classes of total recursive functions. Lecture Notes in Computer Science, Springer, 872, 100-105, 1994.

[6] R. Freivalds, M. Karpinski and C. H. Smith. Co-learning of total recursive functions. Proc. COLT'94, 190-197, 1994.

[7] R. Freivalds, E. B. Kinber and C. H. Smith. On the Impact of Forgetting on Learning Machines. Journal of the ACM, 42(6): 11461168,1995
[8] R. Freivalds, M. Karpinski, C. H. Smith, R. Wiehagen. Learning by the Process of Elimination. Information and Computation, 176(1): 37-50, 2002.

[9] M. Kummer. A learning-theoretic characterization of classes of recursive functions. Information Processing Letters, 54(4): 205-211, 1995.

[10] A. I. Malcev. Algorithms and Recursive Functions. Nauka, Moscow, 1965 (Russian).

[11] P. Odifreddi. Classical Recursion Theory: The Theory of Functions and Sets of Natural Numbers, Vol. 1 (Studies in Logic and the Foundations of Mathematics, Vol. 125), North Holland, 1992.

[12] H. Rogers, Jr. Theory of Recursive Functions and Effective Computability. MIT Press, 1987. 